ARTICLE

\title{
Room temperature near unity spin polarization in 2D Van der Waals heterostructures
}

Danliang Zhang 1 , Ying Liu², Mai He', Ao Zhang³, Shula Chen², Qingjun Tong1, Lanyu Huang1, Zhiyuan Zhou', Weihao Zheng ${ }^{2}$, Mingxing Chen $^{3}$, Kai Braun ${ }^{1,4}$, Alfred J. Meixner ${ }^{4}$, Xiao Wang (1) ${ }^{1 凶} \&$ Anlian Pan (D) ${ }^{2 凶}$

The generation and manipulation of spin polarization at room temperature are essential for 2D van der Waals (vdW) materials-based spin-photonic and spintronic applications. However, most of the high degree polarization is achieved at cryogenic temperatures, where the spin-valley polarization lifetime is increased. Here, we report on room temperature high-spin polarization in 2D layers by reducing its carrier lifetime via the construction of $\mathrm{vdW}$ heterostructures. A near unity degree of polarization is observed in $\mathrm{Pbl}_{2}$ layers with the formation of type-I and type-II band aligned vdW heterostructures with monolayer $\mathrm{WS}_{2}$ and $\mathrm{WSe}_{2}$. We demonstrate that the spin polarization is related to the carrier lifetime and can be manipulated by the layer thickness, temperature, and excitation wavelength. We further elucidate the carrier dynamics and measure the polarization lifetime in these heterostructures. Our work provides a promising approach to achieve room temperature high-spin polarizations, which contribute to spin-photonics applications.

\footnotetext{
${ }^{1}$ Key Laboratory for Micro-Nano Physics and Technology of Hunan Province, School of Physics and Electronics, Hunan University, Changsha 410082, China. ${ }^{2}$ Key Laboratory for Micro-Nano Physics and Technology of Hunan Province, College of Materials Science and Engineering, Hunan University, Changsha 410082, China. ${ }^{3}$ Key Laboratory for Matter Microstructure and Function of Hunan Province, School of Physics and Electronics, Hunan Normal University, Changsha 410081, China. ${ }^{4}$ Institute of Physical and Theoretical Chemistry and LISA+, University of Tübingen, Auf der Morgenstelle 18,72076

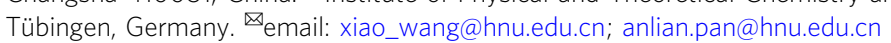


$\mathrm{V}$ an der Waals (vdW) heterostructures formed by different two-dimensional (2D) materials are emerging as an excellent platform for both fundamental research and technical applications ${ }^{1-3}$, due to the unique photonic, optoelectronic properties of the individual materials, and the combination their advantages in heterostructures ${ }^{4-7}$. The band alignment at the interface of the $\mathrm{vdW}$ heterostructures can modulate the interfacial carrier behaviors, which is essential for device applications ${ }^{8}$. Up to now, vdW heterostructure-based logic transistors ${ }^{9}$, modulators ${ }^{10}$, light-emitting diodes (LEDs) ${ }^{11}$, fast photodetectors ${ }^{12}$, and photovoltaic cells ${ }^{13}$ have been realized, which make them important building blocks for photonic and optoelectronic devices and integrated circuits.

With the development of quantum information science, the $2 \mathrm{D}$ materials-based spin-photonic devices are highly desirable. The generation and control of spin polarization is at the heart of this field. One recent progress along this direction is the light-induced spin and valley polarization in transition metal dichalcogenides $(\text { TMDCs) })^{14-17}$ and their heterostructures ${ }^{18-22}$. However, most of these studies were performed at low temperature with highquality samples, so as to reduce the intervalley scattering and increase the spin-valley polarization lifetime ${ }^{23,24}$. It is therefore greatly desirable to find new materials and/or mechanism to obtain a high degree of spin polarization at room temperature, which is essential for practical spintronic applications.

Compared to typical TMDCs such as $\mathrm{WS}_{2}$ and $\mathrm{WSe}_{2}$, layered lead iodine $\left(\mathrm{PbI}_{2}\right)$ has a wider bandgap and higher light absorption coefficient, showing potential optoelectronic applications $^{25-28}$, such as photodetectors ${ }^{29-32}$, X-ray or $\gamma$-ray detection $^{33,34}$, and optically pumped lasers ${ }^{35}$. $\mathrm{PbI}_{2}$ has a hexagonal crystal pattern composed of covalently bonded repeating sequences of I-Pb-I atomic layers, with weak vdW interaction between the two layers ${ }^{25,26}$. The bandgap can be tuned from a direct gap of $2.28 \mathrm{eV}$ to an indirect-gap of $2.63 \mathrm{eV}$ when reducing its thickness or a fine-tuning by applying $\operatorname{strain}^{25,26}$. Due to the $v d W$ nature, layered $\mathrm{PbI}_{2}$ can easily form heterostructures with other TMDCs materials ${ }^{36}$, exhibiting versatile band alignment ${ }^{37,38}$. Furthermore, since $\mathrm{PbI}_{2}$ can be used as a precursor of lead halide perovskite, the conversion from $\mathrm{PbI}_{2} / \mathrm{TMDCs}$ to perovskite/TMDCs heterostructures have been realized ${ }^{39,40}$, which further extends the applications of $\mathrm{PbI}_{2}$. The formation of high-quality heterostructures provides the opportunity to manipulate the carrier dynamics in the vertical direction and affects the carrier lifetime ${ }^{41}$.

Here, we report on room temperature high-spin polarization in $\mathrm{PbI}_{2}$ layers via the construction of heterostructures with monolayer TMDCs. Due to the reduction of carrier lifetime, a nearunity degree of polarization at room temperature is observed from both layered $\mathrm{PbI}_{2} /$ monolayer- $\mathrm{WS}_{2}$ type-I and $\mathrm{PbI}_{2} /$ monolayer-WSe $e_{2}$ type-II band aligned heterostructures. The spin polarization related to the carrier lifetime can be manipulated by the material thickness, temperature, and excitation wavelength, providing versatile control means for further practical applications. In addition, we investigate the polarized carrier dynamics in heterostructures and reveal the polarization lifetime by timeresolved polarization experiments. This work not only provides a basis for controlling carrier dynamics and spin polarization in 2D $\mathrm{vdW}$ heterostructures but also offers a strategy to achieve a high degree of spin polarization at room temperature, promising for spin-photonics applications.

\section{Results}

$\mathrm{PbI}_{2} / \mathrm{WS}_{2}$ heterostructures and near-unity polarization. The heterostructures consisting of a bottom $\mathrm{WS}_{2}$ monolayer and top $\mathrm{PbI}_{2}$ layers with different thicknesses were prepared with a two-step physical vapor deposition (PVD) method. Typical heterostructures show a triangular shape with a uniform and smooth surface (Fig. 1a). Based on the calculated electronic structures from previous works ${ }^{38,41}$, the formed $\mathrm{PbI}_{2} / \mathrm{WS}_{2}$ heterostructures show a type-I band alignment, such that the photogenerated electrons and holes can be transferred from the $\mathrm{PbI}_{2}$ to the $\mathrm{WS}_{2}$ monolayer (Fig. 1a), which is also confirmed by our experimental results. Since circularly polarized photons carry angular momentum components, the absorption of a circularly polarized laser leads to the so-called optical spin injection to the materi$\mathrm{als}^{42}$. Figure $1 \mathrm{~b}$ shows the band structure for the $\mathrm{PbI}_{2}$ thin film with a thickness of 20 layers (details of density-functional theory calculations are given in Supplementary Note 1). Both the valence band maximum (VBM) and conduction band minimum (CBM) locate at the $\Gamma$ point and are spin degenerate because the thin film has the inversion symmetry and time-reversal symmetry. Figure $1 \mathrm{c}$ shows schematically the optical selection rules for $\mathrm{PbI}_{2}$ layers at $\Gamma$ point. The right-handed $(\sigma+)$ polarized photon carrying an angular monument of $+\hbar$ causes the transition from the spin state $-1 / 2$ to $+1 / 2$, while left-handed $(\sigma-)$ excitation referring to the transition from the spin state $+1 / 2$ to $-1 / 2$. The degree of photoluminescence (PL) circular polarization $(\rho)$ can be defined as $\rho_{\mathrm{PL}}=\left(I_{\sigma-/ \sigma+}-I_{\sigma+/ \sigma-}\right) /\left(I_{\sigma+}+I_{\sigma-}\right)$ under $\sigma-/ \sigma+$ polarized excitation, where $I_{\sigma+}$ and $I_{\sigma-}$ denote the $\sigma+$ and $\sigma-$ polarized PL intensities, respectively.

Experimentally observed polarized PL spectra (Fig. 1d) and the calculated $\rho$ (Fig. 1e) from pure $\mathrm{PbI}_{2}$ layers (grown on $\mathrm{SiO}_{2} / \mathrm{Si}$ substrate, about $100 \mathrm{~nm}$ ) show a small $(\rho \approx 0.1$ for $488 \mathrm{~nm}$ excitation) degree of polarization and also an excitation wavelength dependence that further away from the resonance excitation leads to a much smaller value (Supplementary Fig. 5). Such a low polarization can be due to the spin relaxation during the carrier lifetime. Considering the carrier lifetime $\left(\tau_{c}\right)$ and spin relaxation time $\left(\tau_{s}\right)$, the measured degree of the polarization can be expressed as $P=P_{0} /\left(1+\tau_{\mathrm{c}} / \tau_{\mathrm{s}}\right)$, where $P_{0}$ is the polarization without spin relaxation ${ }^{42}$. For carrier lifetimes much longer than the spin relaxation $\left(\tau_{c} » \tau_{\mathrm{s}}\right)$, the polarization will be small. In order to obtain a high polarization of $P$, one should either increase the spin relaxation time $\left(\tau_{\mathrm{s}}\right)$ or decrease the carrier lifetime $\left(\tau_{c}\right)$. Although the former depends on the detailed material and experiment conditions and thus is hard to control, the latter can be engineered by stacking different $2 \mathrm{D}$ materials to form a vdW heterostructure. The mechanism behind is that in a $\mathrm{vdW}$ heterostructure the band alignment leads to interlayer charge transfer that would significantly reduce the carrier lifetime and thus increase the spin polarization. For pure $\mathrm{WS}_{2}$ monolayer, the circular polarization excitation creates the valley polarization and the degree of polarization is in general small under off-resonance excitation at room temperature (Supplementary Fig. 6) ${ }^{43}$.

The $\mathrm{PbI}_{2} / \mathrm{WS}_{2}$ heterostructures were excited with $\sigma-$ excitation and the PL emission with $\sigma+$ and $\sigma-$ polarizations were detected (Fig. 1f). For the PL signal from $\mathrm{PbI}_{2}$, we observe that the dominating emission is $\sigma-$ polarized (black). Compared to the pure $\mathrm{PbI}_{2}$ layers, the degreed of polarization of $\mathrm{PbI}_{2}$ in heterostructures increases dramatically, almost reaching $100 \%$ at room temperature (Fig. $1 \mathrm{~g}$ ), consistent with our above mechanism. Explicitly, the carrier transfer process dramatically reduces the carrier lifetime of $\mathrm{PbI}_{2}{ }^{41}$, which leads to a small value of $\tau_{\mathrm{c}} / \tau_{\mathrm{s}}$ hence resulting in near-unity spin polarizations under resonance excitation. In contrast, the degree of polarization of the $\mathrm{WS}_{2}$ monolayer in heterostructures decreases, showing almost no polarization. Therefore, $\mathrm{vdW}$ heterostructures, where extra decay channels reduce the carrier lifetime largely, provide an excellent platform to achieve a high degree of polarization. 
a

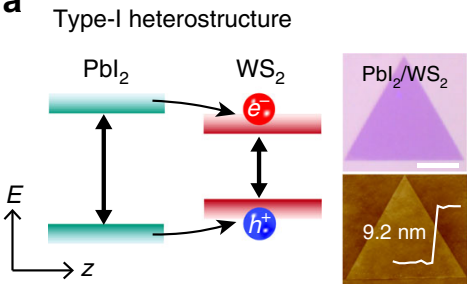

d

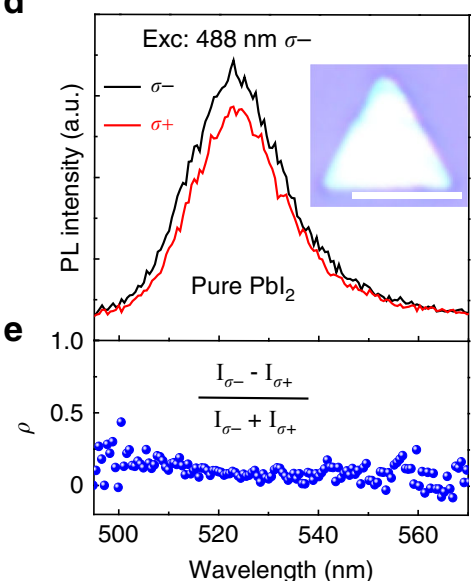

b

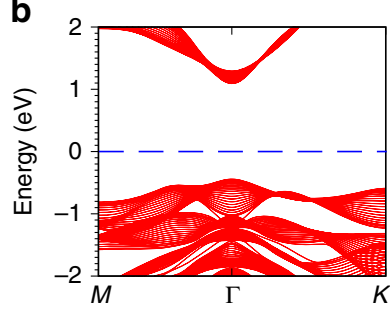

C $\quad \mathrm{Pbl}_{2}$

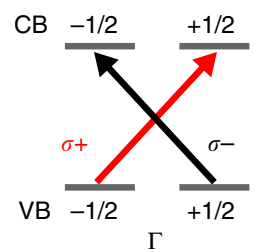

f

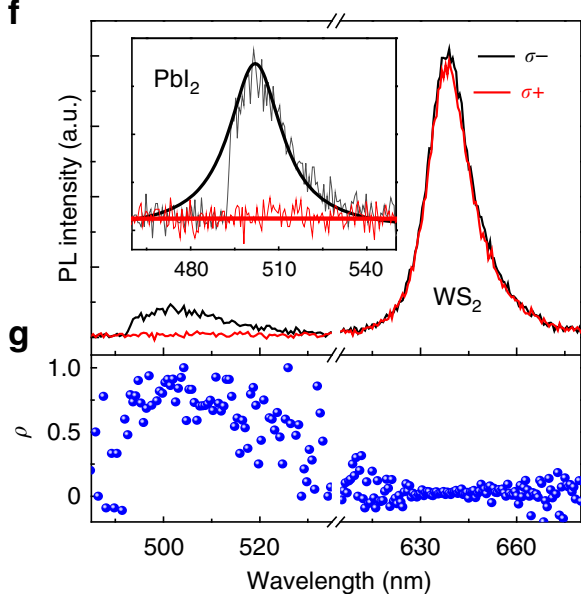

Fig. 1 Spin polarization mechanism and spectrum of $\mathbf{P b l}_{\mathbf{2}} / \mathbf{W S}_{\mathbf{2}}$ heterostructures. a Schematic of the type-I heterostructure for the study of the carrier interlayer transportation. Both electrons and holes transfer from $\mathrm{Pbl}_{2}$ to $\mathrm{WS}_{2}$. The images on the right are optical and $\mathrm{AFM}$ micrographs of a Pbl$/ 2 / \mathrm{WS}_{2}$ heterostructure with a thickness of $9.2 \mathrm{~nm}$. b Band structure for the $\mathrm{Pbl}_{2}$ thin film with a thickness of 20 layers, which is spin degenerate. c Schematic illustration of the polarized optical transitions in $\mathrm{Pbl}_{2}$ thin films. d Circularly polarized $\mathrm{PL}$ spectra from pure $\mathrm{Pbl}_{2}$ at room temperature. e Corresponding

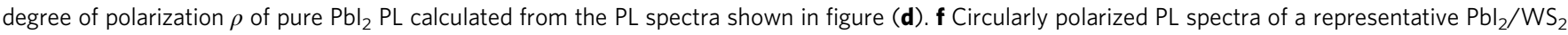
heterostructure at room temperature. $\mathbf{g}$ Corresponding degree of polarization $\rho$ calculated from the PL spectra shown in figure (f). All length of the scale bar is $10 \mu \mathrm{m}$.

To further verify the carrier lifetime enhanced the degree of polarization, we investigated how it depends on the thickness of the $\mathrm{PbI}_{2}$ layer while maintaining the bottom monolayer $\mathrm{WS}_{2}$ in the heterostructures. For pure $\mathrm{PbI}_{2}$ layers with different thicknesses, the PL shows similar decay curves in time-resolved PL (TRPL) spectra (Supplementary Fig. 7). In contrast, increasing the thickness of the $\mathrm{PbI}_{2}$ layer in heterostructures will increase its carrier lifetime, which would decrease the obtained polarization. Figure 2 displays the circularly polarized PL spectra from $\mathrm{PbI}_{2} /$ $\mathrm{WS}_{2}$ heterostructures with the total thickness varying from 9.2 to $22.6 \mathrm{~nm}$, with $\sigma-$ excitation. For the PL signal from $\mathrm{PbI}_{2}$ (Fig. 2a), besides the overall dominating emission with $\sigma-$ polarization (black), we observe that the weak emission signal from the $\sigma+$ polarization (red) increases with the increasing thickness of heterostructures. In contrast, the relative intensities of $\sigma+$ and $\sigma-$ emission from the monolayer $\mathrm{WS}_{2}$ in the heterostructures are almost unchanged (Fig. 2b), showing the small degree of polarization as we discussed previously. With the spectra in Fig. 2a, we have calculated $\rho$ for the $\mathrm{PbI}_{2}$ PL from different heterostructures and show that $\rho$ decreases from 0.998 to 0.693 as the heterostructures thickness increases from 9.2 to $22.6 \mathrm{~nm}$ (Fig. 2c).

This phenomenon is related to the changing of carrier lifetime and can be understood by following the equation $P(X)=P_{0} /(1+$ $\tau_{\text {eff }(X)} / \tau_{s}$ ), where $\tau_{\text {eff }}$ is the effective lifetime of the carriers, $X$ is the thickness of the $\mathrm{PbI}_{2}$ layers (Supplementary Note 2). Based on the coupled rate equations of spin injected carriers, we performed simulations of the degree of polarization for $\mathrm{PbI}_{2} \mathrm{PL}$ in heterostructures (solid line in Fig. 2c) as a function of heterostructure thickness. One can see that the simulations are in good agreement with our experimental data (solid spheres in Fig. 2c). We illustrate the phenomena in Fig. 2d, e for typical thin and thicker $\mathrm{PbI}_{2}$ in heterostructures that the spin-flip within the longer carrier lifetime leads to a smaller degree of polarization. We also measured excitation power dependent circularly polarized PL spectra, and reveal only a slight decrease of the $\rho$ of $\mathrm{PbI}_{2} \mathrm{PL}$ when the excitation power increases from 2 to $10 \mu \mathrm{W}$ (Supplementary Fig. 8).

Polarization dynamics. To probe the polarization lifetime, we have excited a $13.6 \mathrm{~nm}$ thick $\mathrm{PbI}_{2} / \mathrm{WS}_{2}$ heterostructure with $\sigma+$ polarized $100-\mathrm{fs}$ laser at $441 \mathrm{~nm}$, and recorded the spectrally integrated and time-resolved $\sigma+$ and $\sigma-$ PL emission, respectively. The integrated PL intensity (Fig. 3a) shows a degree of polarization of about 0.3 for $\mathrm{PbI}_{2}$, and almost zero degree of polarization for $\mathrm{WS}_{2}$ (Fig. 3c). Compared with the experiments under the excitation at $488 \mathrm{~nm}$, we observed a reduced degree of polarization due to the off-resonance excitation, which is similar to the observations of many TMDCs atomic layers under different excitation photon energies ${ }^{14,44}$. Almost no polarization can be observed for the same heterostructures exited with $100 \mathrm{fs}$ pulsed laser at $400 \mathrm{~nm}$, since they are even further off-resonance (Supplementary Fig. 9). In Fig. 3b, we show the $\sigma+$ (black) and $\sigma-(\mathrm{red}) \mathrm{PbI}_{2} \mathrm{PL}$ emission decay detected in the spectral range from 2.30 to $2.53 \mathrm{eV}$ by a streak camera. The dashed line represents the instrument response function (IRF) obtained from pure laser pulses under the same experimental condition. After the deconvolution of the measured decay curves from the IRF and the fitting, we obtain the decay times for the $\sigma+$ and $\sigma$ - polarized $\mathrm{PbI}_{2} \mathrm{PL}$ emission, which are 15.7 and 27.5 ps, respectively. With 


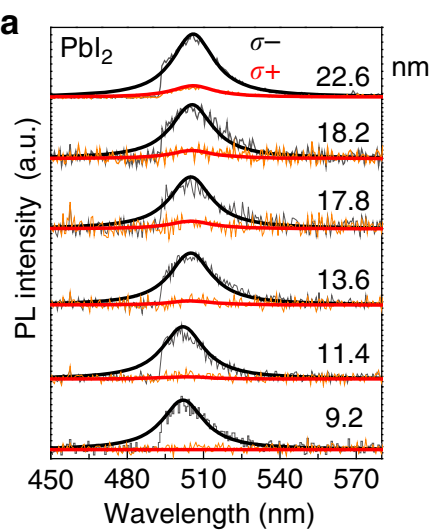

d

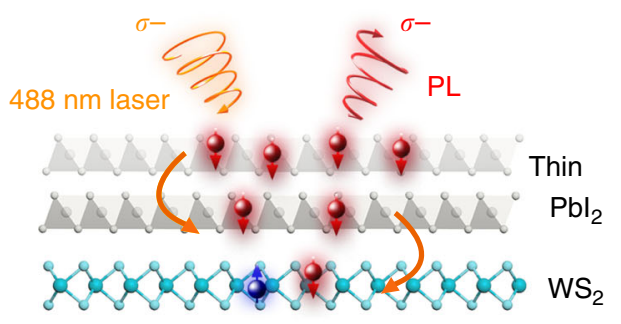

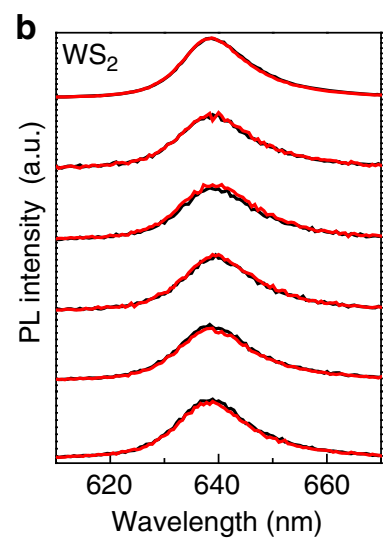

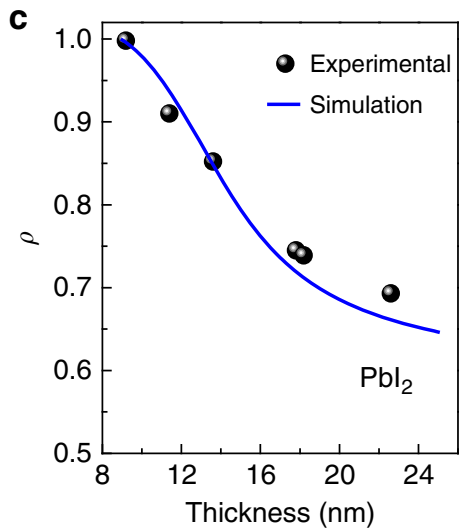

e

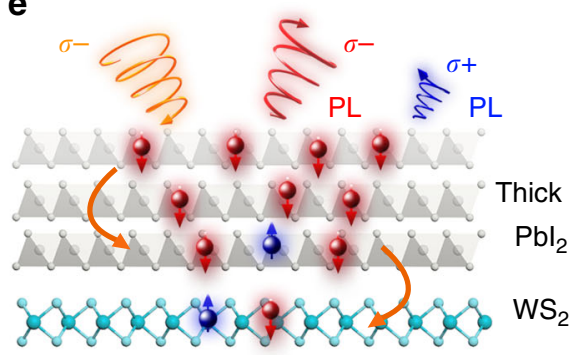

Fig. 2 Thickness-dependent spin polarization and the underlying mechanism in $\mathbf{P b} \mathbf{b}_{\mathbf{2}} / \mathbf{W} \mathbf{S}_{\mathbf{2}}$ heterostructures. $\mathbf{a}$, $\mathbf{b}$ Circularly polarized $\mathrm{PL}$ spectra of $\mathrm{Pbl}{ }_{2}$ and $\mathrm{WS}_{2}$ in $\mathrm{Pbl}_{2} / \mathrm{WS}_{2}$ heterostructures with different thicknesses. c Degree of polarization $\rho$ of $\mathrm{Pbl}_{2}$ as a function of thickness of the heterostructures. The solid line represents the numerical simulation result. $\mathbf{d}$, e Schematic illustrations of the resulting spin polarization in the thin and thick $\mathrm{Pbl}_{2} / \mathrm{WS}_{2}$ heterostructures. The curled curves represent the incident light and the PL emissions. The red balls represent spin-down electrons, and the blue balls represent spin-up electrons.
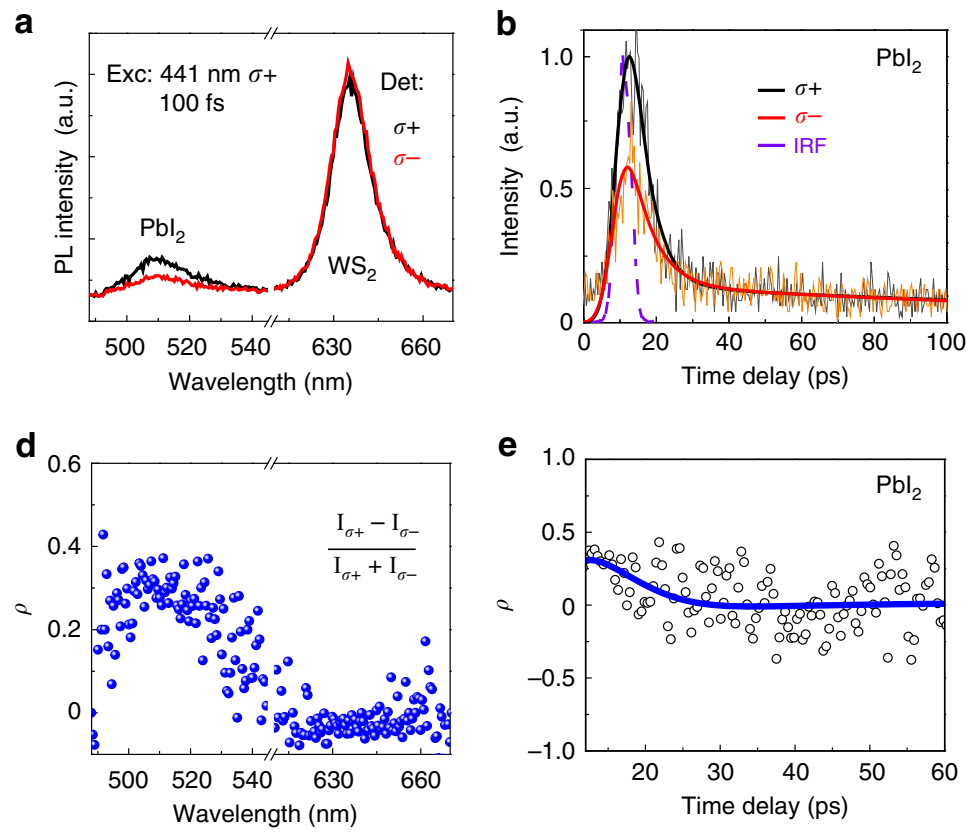

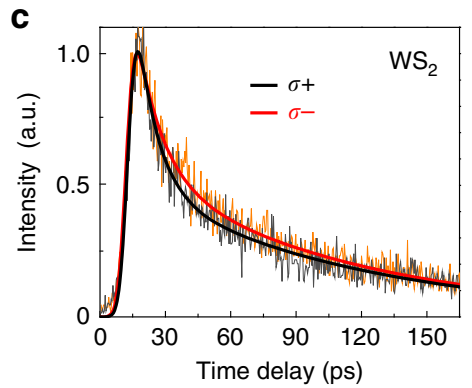

Fig. 3 Spin polarization dynamics of $\mathbf{P b l}_{\mathbf{2}} / \mathbf{W S}_{\mathbf{2}}$ heterostructures. a-c Circularly polarized PL spectra (a) and TRPL data from Pbl 2 (b), and WS $\mathrm{CS}_{2}(\mathbf{c})$ of $\mathrm{Pbl}_{2} / \mathrm{WS}_{2}$ heterostructures. $\mathbf{d}-\mathbf{f}$ Corresponding degree of polarization $\rho$ as a function of wavelength and time calculated from the PL and TRPL spectra. For excitation, a $441 \mathrm{~nm} \sigma+$ polarized fs-pulsed laser beam was used.

these two time-resolved polarized PL decays, we can calculate the degree of polarization as a function of time, revealing the polarization lifetime of about 15 ps (Fig. 3e). For resonance excitation, the polarization lifetime is expected to be longer ${ }^{45}$, which could lead to a higher degree of polarization due to a smaller $\tau_{\mathrm{c}} / \tau_{\mathrm{s}}$. For the $\mathrm{WS}_{2}$ monolayer in the heterostructures, the $\sigma+$ and $\sigma-$ polarized PL emissions show similar decay curves of about 49 ps (Fig. 3c), consistent with the integrated PL spectra, leading to the non-observable polarization lifetime (Fig. 3f). It should be noted that the dominating excited carriers account for the $\mathrm{WS}_{2}$ $\mathrm{PL}$ in heterostructures originate from the transferred carriers, not the optical valley initialization in pure $\mathrm{WS}_{2}$ monolayer. 

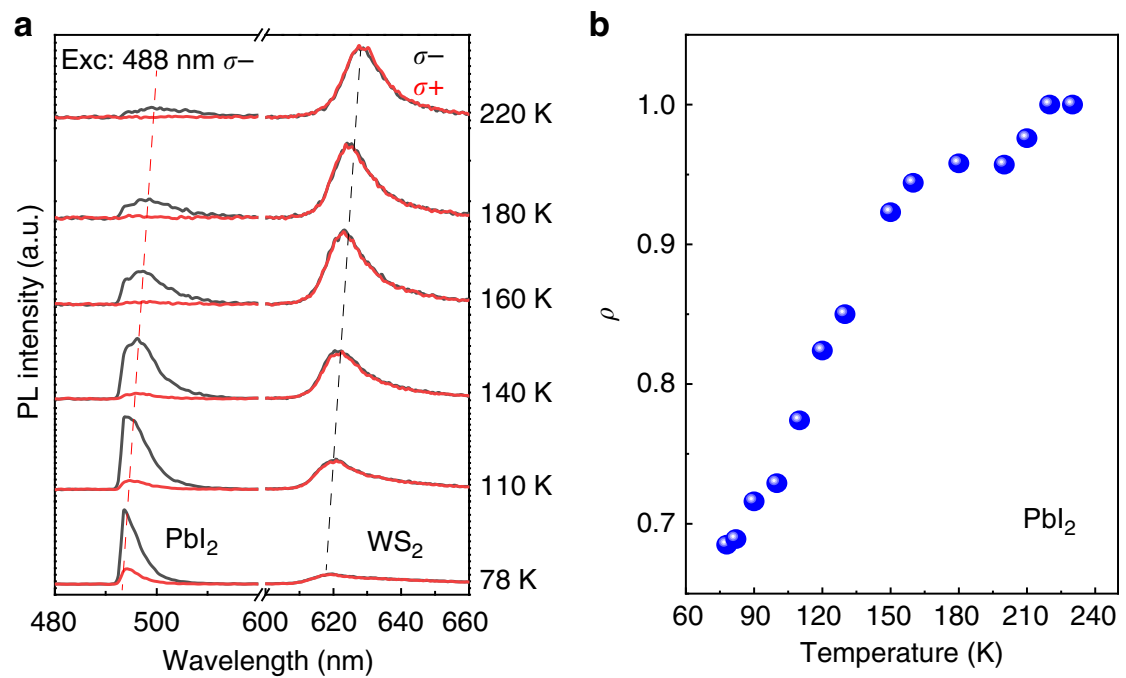

Fig. 4 Temperature-dependent degree of polarization from $\mathbf{P b l}_{\mathbf{2}} / \mathbf{W S}_{\mathbf{2}}$ heterostructures. a Circularly polarized $\mathrm{PL}$ spectra of $\mathrm{Pbl}_{2} / \mathrm{WS}_{2}$ heterostructure at different temperatures for excitation with a $488 \mathrm{~nm} \sigma$ - polarized CW laser beam. b Degree of polarization $\rho$ of $\mathrm{PL} \mathrm{from} \mathrm{Pbl} 2$ as a function of temperature, showing a decreasing tendency at lower temperatures.

Temperature-dependent polarization. Figure 4a shows the temperature-dependent circularly polarized PL spectra of $\mathrm{PbI}_{2} /$ $\mathrm{WS}_{2}$ heterostructures. With decreasing temperature, the $\mathrm{PL}$ emission from $\mathrm{PbI}_{2}$ and $\mathrm{WS}_{2}$ blue shifts, as indicated by the dashed line. The PL intensity of $\mathrm{PbI}_{2}$ largely increases in comparison with that of $\mathrm{WS}_{2}$. This is because the interlayer charge transfer from $\mathrm{PbI}_{2}$ to $\mathrm{WS}_{2}$ is momentum mismatched. This process is only allowed with the assistance of phonons, which is suppressed greatly at low temperatures. As a result, the carrier lifetime in the $\mathrm{PbI}_{2}$ layer is increased with a decrease in temperature, similar to the effect of increasing layer thickness, which leads to a decrease in the degree of polarization $\rho$. Indeed, we found $\rho$ of $\mathrm{PbI}_{2} \mathrm{PL}$ in heterostructures decreases from 0.99 to 0.68 when the temperature decreases from 220 to $78 \mathrm{~K}$ (Fig. 4b). This temperature dependence is in contrast to the case for the pure TMDCs, where the degree of polarization in TMDCs monolayer normally increases at lower temperatures due to the reduced intervalley scattering. In control measurements with pure $\mathrm{PbI}_{2}$ layers, we find that the small $\rho$ at room temperature slightly increases at low temperatures (Supplementary Fig. 11), which indicates that the observed temperature-dependent polarization in heterostructures does not originate from the intrinsic property of pure $\mathrm{PbI}_{2}$ but due to interlayer charge transfer process. Our results suggest that $\mathrm{PbI}_{2}$ in $\mathrm{PbI}_{2} / \mathrm{WS}_{2}$ heterostructures shows a higher polarization at room temperature, which makes them even more practical for device applications.

Near-unity polarization from $\mathrm{PbI}_{2} / \mathrm{WSe}_{2}$ heterostructures. We have studied the degree of polarization in the PL spectra emitted from $\mathrm{PbI}_{2} / \mathrm{WSe}_{2}$ heterostructures, which have the type-II band alignment that photogenerated electrons can transfer from $\mathrm{WSe}_{2}$ to $\mathrm{PbI}_{2}$ and holes transfer from $\mathrm{PbI}_{2}$ to $\mathrm{WSe}_{2}$ (Fig. 5a). The formation of type-II alignment also reduces the carrier lifetime in $\mathrm{PbI}_{2}$, due to the extra decay channel ${ }^{38}$. Therefore, a high degree of polarization is expected for $\mathrm{PbI}_{2} \mathrm{PL}$ in $\mathrm{PbI}_{2} / \mathrm{WSe}_{2}$. Figure $5 \mathrm{~b}$ shows the circular polarization-resolved PL emission of a $9.5-\mathrm{nm}$ thick heterostructure, under $\sigma-$ polarized continuous-wave (CW) laser excitation at $488 \mathrm{~nm}$. For $\mathrm{PbI}_{2} \mathrm{PL}$ emission, we observe that $\sigma-$ polarization (black) dominates the whole emission. The calculated degree of polarization (Fig. 5b, lower panel) approaches $100 \%$ at room temperature, which is due to the largely reduced carrier lifetime with a very small $\tau_{\mathrm{c}} / \tau_{\mathrm{s}}$. Similar to $\mathrm{PbI}_{2} / \mathrm{WS}_{2}$ heterostructures, the thicker heterostructures show a slightly smaller polarization value (Supplementary Fig. 12). The valley polarization of $\mathrm{WSe}_{2}$ is very low because the excitation light is far from resonance, even though its carrier lifetime reduces as well. We further performed time-resolved $\sigma+$ and $\sigma-$ polarized PL emission experiments under the excitation of $\sigma+$ polarized $100-\mathrm{fs}$ laser at $441 \mathrm{~nm}$. The integrated PL intensity shows a degree of polarization of about 0.3 for $\mathrm{PbI}_{2}$, and almost zero degree of polarization for $\mathrm{WSe}_{2}$ (Fig. 5c). After the deconvolution of the measured decay curve from the IRF and the fitting (Fig. 5d), we obtain the $\sigma+$ and $\sigma-$ polarized PL emission with 7.5 and $9.44 \mathrm{ps}$ lifetime, and the polarization lifetime of $13 \mathrm{ps}$ in the $\mathrm{PbI}_{2} / \mathrm{WSe}_{2}$ heterostructure. Considering the smaller value of $P_{0}$ under nonresonance excitation, the obtained $\tau_{\mathrm{c}}$ and $\tau_{\mathrm{s}}$ is general in agreement with the observed degree of polarization. For $\mathrm{WSe}_{2}$, the $\sigma+$ and $\sigma-$ polarized PL emissions show similar decay curves of about 30 ps (Fig. 5e), showing a non-observable polarization lifetime, which agrees with the integrated PL spectra and the close to zero degree of polarization. With temperature-dependent polarization measurements, we find that the $\rho$ of $\mathrm{PbI}_{2} \mathrm{PL}$ decreases with the temperature decreases (Supplementary Fig. 13), which is consistent with the temperature dependence in $\mathrm{PbI}_{2} / \mathrm{WS}_{2}$ heterostructure.

\section{Discussion}

In conclusion, we have demonstrated the realization of a nearunity spin polarization in layered $\mathrm{PbI}_{2} / \mathrm{WS}_{2}$ and $\mathrm{PbI}_{2} / \mathrm{WSe}_{2}$ in $\mathrm{vdW}$ heterostructures at room temperature via $\mathrm{vdW}$ engineering. The underlying physics relies on the reduction of the carrier lifetime through interlayer carrier transfer in vdW heterostructures. We observe a thickness-dependent degree of polarization of $\mathrm{PbI}_{2}$ in $\mathrm{PbI}_{2} / \mathrm{WS}_{2}$ heterostructures, that the thicker the $\mathrm{PbI}_{2}$ layers the smaller the degree of polarization. By timeresolved circulary polarization measurements, we reveal the spin polarization lifetime of $\mathrm{PbI}_{2}$ in heterostructures. The degree of polarization is also found to have an excitation power and wavelength dependence. The higher the excitation power leads to a slight decrease in the polarization. For excitations with photon energy away from the resonance, we observed a smaller polarization. The polarization decreases to zero under the excitation of $400 \mathrm{~nm}$ laser. Furthermore, we also demonstrated that the nearunity degree of polarization can be obtained in a $\mathrm{PbI}_{2}$ and 
a

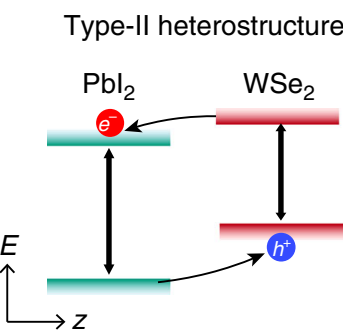

b

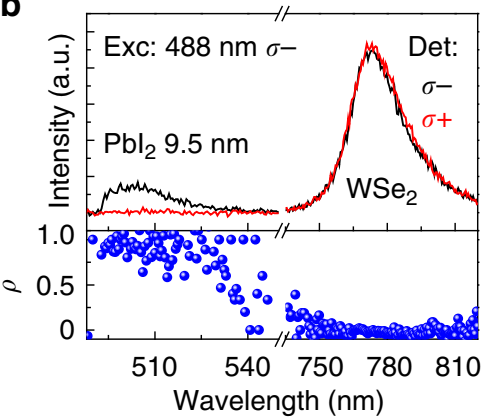

C

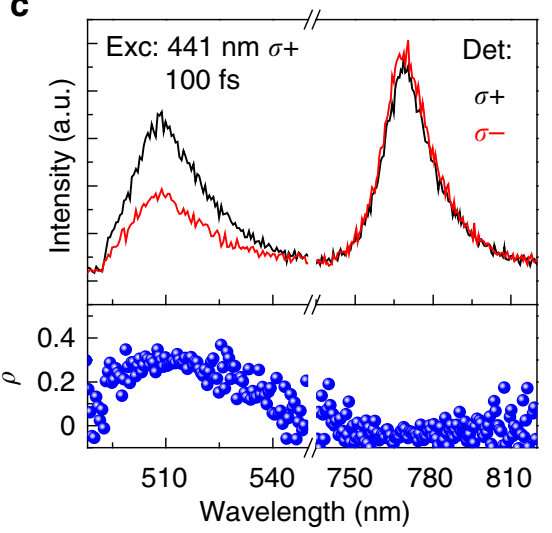

d
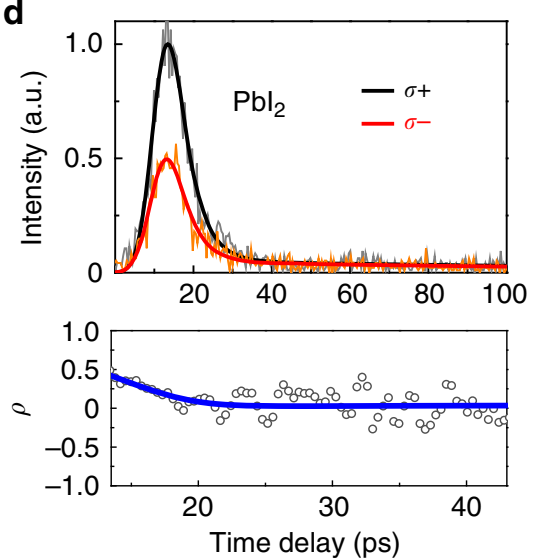
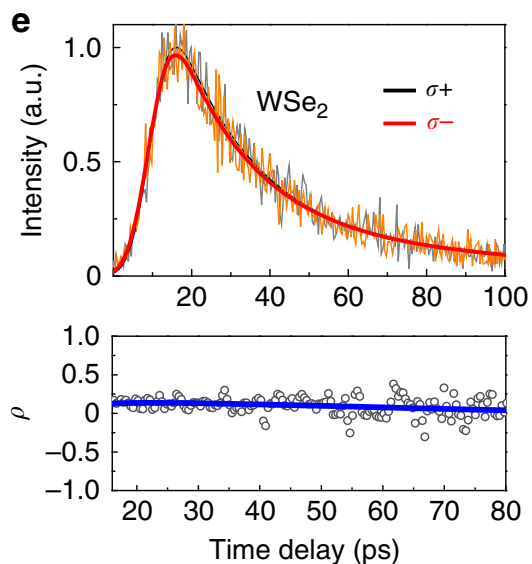

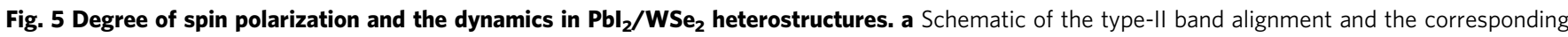
photogenerated carrier behaviors in $\mathrm{Pbl}_{2} / \mathrm{WSe}_{2}$ heterostructures. b Circularly polarized PL spectra from a $9.5 \mathrm{~nm}$ thick $\mathrm{Pbl}_{2} / \mathrm{WSe}_{2}$ heterostructure, and the corresponding calculated $\rho$ as a function of wavelength. The excitation is $488 \mathrm{~nm} \sigma$ - polarized CW laser. c-e Circularly polarized PL spectra (c) and TRPL data from $\mathrm{Pbl}_{2}$ (d) and $\mathrm{WSe}_{2}(\mathbf{e})$ in $\mathrm{Pbl}_{2} / \mathrm{WSe} e_{2}$ heterostructure, and the corresponding degree of polarization $\rho$ calculated from PL and TRPL spectra. Excitation with $441 \mathrm{~nm} \sigma+$ polarization femtosecond laser pulses.

monolayer-WSe $\mathrm{S}_{2}$ heterostructure with a type-II band alignment, which can also be understood by manipulating the carrier lifetime.

The realization of high-spin polarization via the formation of heterostructure in principle can be applied with other substrates that quenches the PL. However, one needs to consider the balance between the increasing degree of polarization and the PL quenching. For $\mathrm{PbI}_{2} / \mathrm{TMDCs}$ heterostructures, the $\mathrm{PL}$ in $\mathrm{PbI}_{2}$ is quenched about one order of magnitude but the remaining intensity is still comparable with that of typical monolayer TMDCs. On the other hand, monolayer TMDCs provide an excellent platform to construct a high-quality heterostructure with $\mathrm{PbI}_{2}$ through vdW interaction, facilitates the efficient transport of carriers.

Moreover, for future applications, not only the high-spin polarization, but also spin coherence are of great importance. The detailed study of the spin coherence should take into account the effect of the temperature-related dephasing and is an interesting topic for future study. Our study provides a promising strategy via $\mathrm{vdW}$ engineering to achieve a high degree of polarization in vdW $2 \mathrm{D}$ materials at room temperature, which could provide valuable information for spintronics.

\section{Methods}

Sample preparation. The $\mathrm{PbI}_{2} / \mathrm{TMDCs}$ heterostructures were synthesized by a two-step PVD method. Briefly, monolayer TMDCs $\left(\mathrm{WS}_{2}\right.$, $\left.\mathrm{WSe}_{2}\right)$ serving as the bottom layers for the heterostructures were synthesized first by PVD method. In the second step, lead iodide powder was placed in the central area of the tube furnace, the substrates with grown monolayer TMDCs from the first step were placed in the downstream area of the tube furnace. The pressure in the furnace was drawn to 40 mtorr by a vacuum pump and the argon flow rate was maintained at $20 \mathrm{sccm}$. The heating lasts $10 \mathrm{~min}$ at a temperature of $400^{\circ} \mathrm{C}$. By varying the deposition temperature of $\mathrm{PbI}_{2}$ from 180 to $220^{\circ} \mathrm{C}$, we obtained a growth trend of thin to thick $\mathrm{PbI}_{2} / \mathrm{TMDCs}$ heterostructures. The thicknesses of the heterostructures were measured with an atomic force microscope (AFM). By the same experimental method, pure $\mathrm{PbI}_{2}$ layers can be grown on $\mathrm{SiO}_{2} / \mathrm{Si}$ or mica substrates. In order to improve the environmental stability of the prepared $\mathrm{PbI}_{2} / \mathrm{WS}_{2}$ and $\mathrm{PbI}_{2} / \mathrm{WSe}_{2}$ heterostructures in optical measurement, all samples were encapsulated with polydimethylsiloxane (PDMS).

Steady-state circular polarization-resolved PL spectroscopy. Steady-state circular polarization-resolved PL measurements were conducted using a confocal microscope (WITec, alpha-300) equipped with a $\times 50$ objective (Zeiss, $0.75 \mathrm{NA}$ ). To create the circularly polarized excitation light source, the laser beam first passes through a linear polarizer (GTH10M-AM-A, Thorlabs), and then a quarter-wave plate (AQWP05M-600, Thorlabs). The circularly polarized beam is reflected by a cube beam splitter (CCM1-BS013/M, Thorlabs) and focused on the sample. The signal is collected by the same objective and is detected by a spectrometer (UHTS300) after passing through a razor edge long pass $488 \mathrm{~nm}$ laser filter, a quarter-wave plate and a polarizer (WP25M-UB, Thorlabs). Left-handed and righthanded PL components of the signal are distinguished by rotating the polarizer. Continuous-wave Ar ion laser (Coherent, Sapphire LP) at $488 \mathrm{~nm}$ (excitation power of $2 \mu \mathrm{W}$ ) is normally used as the excitation source. For comparison, $400 \mathrm{~nm}$ and $441 \mathrm{~nm}$ fs lasers pulses (excitation power of about $20 \mu \mathrm{W}$ ) were used as well. During the experiment, 150 grooves/mm gratings were used for PL measurements.

Low temperature circular polarization-resolved PL measurement. The circularly polarized light is focused by a long working distance objective lens $(\times 50$, Zeiss, NA 0.55) onto the sample located in the cryostat (ST-500, Janis Research Company). The method of circular polarization-resolved PL measurement is the same as that at room temperature.

Dynamics of circular polarization-resolved PL. The dynamics of circular polarization-resolved PL of heterostructures were measured using the same optical microscope (WITec, alpha-300) equipped with a streak camera (C10910, Hamamatsu). A mode-locked Ti: sapphire laser (Tsunami 3941-X1BB, Spectral Physics) (pulse width $100 \mathrm{fs}$, repetition rate $80 \mathrm{MHz}$ ) was used as the fundamental excitation source. Photons at 400 and $441 \mathrm{~nm}$ were generated via the frequency doubling 
from the fundamental 800 and $882 \mathrm{~nm}$ with a BBO crystal. The same optical paths were used to generate circularly polarized 400 and $441 \mathrm{~nm}$ excitation beams. The left- and right-handed circular polarization PL were guided into the streak camera for time-resolved measurements, which were distinguished by the same optical system described for the steady-state measurements.

\section{Data availability}

The data that support the findings of this study are available from the corresponding author upon reasonable request.

Received: 31 January 2020; Accepted: 28 July 2020;

Published online: 07 September 2020

\section{References}

1. Geim, A. K. \& Grigorieva, I. V. Van der Waals Heterostructures. Nature 499, 419-425 (2013).

2. Liu, Y. et al. Van der Waals heterostructures and devices. Nat. Rev. Mater. 1, 16042 (2016)

3. Jariwala, D., Marks, T. J. \& Hersam, M. C. Mixed-dimensional van der Waals heterostructures. Nat. Mater. 16, 170-181 (2017).

4. Wang, Q. H., Kalantar-Zadeh, K., Kis, A., Coleman, J. N. \& Strano, M. S. Electronics and optoelectronics of two-dimensional transition metal dichalcogenides. Nat. Nanotechnol. 7, 699-712 (2012).

5. Mak, K. F. \& Shan, J. Photonics and optoelectronics of 2D semiconductor transition metal dichalcogenides. Nat. Photon. 10, 216-226 (2016).

6. Xia, F., Wang, H., Xiao, D., Dubey, M. \& Ramasubramaniam, A. Twodimensional material nanophotonics. Nat. Photon. 8, 899-907 (2014).

7. $\mathrm{Xu}, \mathrm{W}$. et al. Correlated fluorescence blinking in two-dimensional semiconductor heterostructures. Nature 541, 62-67 (2017).

8. Jin, C. et al. Ultrafast dynamics in van der Waals heterostructures. Nat. Nanotechnol. 13, 994-1003 (2018).

9. Yu, W. J. et al. Vertically stacked multi-heterostructures of layered materials for logic transistors and complementary inverters. Nat. Mater. 12, 246-252 (2013).

10. Sun, Z., Martinez, A. \& Wang, F. Optical modulators with 2 D layered materials. Nat. Photon. 10, 227-238 (2016)

11. Cheng, R. et al. Electroluminescence and photocurrent generation from atomically sharp $\mathrm{WSe}_{2} / \mathrm{MoS}_{2}$ heterojunction $\mathrm{p}-\mathrm{n}$ diodes. Nano Lett. 14 5590-5597 (2014).

12. Massicotte, M. et al. Picosecond photoresponse in van der Waals heterostructures. Nat. Nanotechnol. 11, 42-46 (2016).

13. Lee, C.-H. et al. Atomically thin $\mathrm{p}-\mathrm{n}$ junctions with van der Waals heterointerfaces. Nat. Nanotechnol. 9, 676-681 (2014).

14. Mak, K. F., He, K., Shan, J. \& Heinz, T. F. Control of valley polarization in monolayer $\mathrm{MoS}_{2}$ by optical helicity. Nat. Nanotechnol. 7, 494-498 (2012).

15. Jones, A. M. et al. Optical generation of excitonic valley coherence in monolayer $\mathrm{WSe}_{2}$. Nat. Nanotechnol. 8, 634-638 (2013).

16. Zhang, Y. J., Oka, T., Suzuki, R., Ye, J. T. \& Iwasa, Y. Electrically switchable chiral light-emitting transistor. Science 344, 725-728 (2014).

17. Jiang, C. et al. Zeeman splitting via spin-valley-layer coupling in bilayer $\mathrm{MoTe}_{2}$. Nat. Commun. 8, 802 (2017)

18. Rivera, P. et al. Valley-polarized exciton dynamics in a $2 \mathrm{D}$ semiconductor heterostructure. Science 351, 688-691 (2016).

19. Schaibley, J. R. et al. Directional interlayer spin-valley transfer in twodimensional heterostructures. Nat. Commun. 7, 13747 (2016).

20. Zhang, J. et al. Enhancing and controlling valley magnetic response in $\mathrm{MoS}_{2} / \mathrm{WS}_{2}$ heterostructures by all-optical route. Nat. Commun. 10, 4226 (2019).

21. Zhong, D. et al. Layer-resolved magnetic proximity effect in van der Waals heterostructures. Nat. Nanotechnol. 15, 187-191 (2020).

22. Lorchat et al. Room-temperature valley polarization and coherence in transition metal dichalcogenide-graphene van der Waals heterostructures. ACS Photonics 5, 5047-5054 (2018).

23. Lagarde, D. et al. Carrier and polarization dynamics in monolayer $\mathrm{MoS}_{2}$. Phys. Rev. Lett. 112, 047401 (2014).

24. Kim et al. Observation of ultralong valley lifetime in $\mathrm{WSe}_{2} / \mathrm{MoS}_{2}$ heterostructures. Sci. Adv. 3, e1700518 (2017).

25. Zhong, $\mathrm{M}$. et al. Large-scale $2 \mathrm{D} \mathrm{PbI}_{2}$ monolayers: experimental realization and their indirect band-gap related properties. Nanoscale 9 , 3736-3741 (2017).

26. Yagmurcukardes, M., Peeters, F. M. \& Sahin, H. Electronic and vibrational properties of $\mathrm{PbI}_{2}$ : from bulk to monolayer. Phys. Rev. B 98, 085431 (2018)

27. Sinha, S. et al. Atomic structure and defect dynamics of monolayer lead iodide nanodisks with epitaxial alignment on graphene. Nat. Commun. 11, 1-13 (2020).
28. Cabana, L. et al. Synthesis of $\mathrm{Pbl}_{2}$ single-layered inorganic nanotubes encapsulated within carbon nanotubes. Adv. Mater. 26, 2016-2021 (2014).

29. Wang, Y., Gan, L., Chen, J., Yang, R. \& Zhai, T. Achieving highly uniform two-dimensional $\mathrm{PbI}_{2}$ flakes for photodetectors via space confined physical vapor deposition. Sci. Bull. 62, 1654-1662 (2017).

30. Zheng, W. et al. High-crystalline $2 \mathrm{D}$ layered $\mathrm{PbI}_{2}$ with ultrasmooth surface: liquid-phase synthesis and application of high-speed photon detection. Adv Electron. Mater. 2, 1600291 (2016).

31. Zhang, J. et al. Low-temperature heteroepitaxy of $2 \mathrm{D} \mathrm{PbI}_{2} /$ graphene for largearea flexible photodetectors. Adv. Mater. 30, 1803194 (2018).

32. Xiao, H., Liang, T. \& Xu, M. Growth of ultraflat $\mathrm{PbI}_{2}$ nanoflakes by solvent evaporation suppression for high-performance UV photodetectors. Small 15, 1901767 (2019).

33. Zhu, X. et al. Comparison of electrical properties of X-ray detector based on $\mathrm{PbI}_{2}$ crystal with different bias electric field configuration. J. Mater. Sci. Mater. Electron 27, 11798-11803 (2016).

34. Zhu, X. H., Wei, Z. R., Jin, Y. R. \& Xiang, A. P. Growth and characterization of a $\mathrm{PbI}_{2}$ Single crystal used for gamma ray detectors. Cryst. Res. Technol. 42, 456-459 (2007).

35. Liu, X. et al. Whispering gallery mode lasing from hexagonal shaped layered lead iodide crystals. ACS Nano 9, 687-695 (2015).

36. Xiao, J. et al. $\mathrm{PbI}_{2}-\mathrm{MoS}_{2}$ heterojunction: van der Waals epitaxial growth and energy band alignment. J. Phys. Chem. Lett. 10, 4203-4208 (2019).

37. Sun, Y. et al. Band structure engineering of interfacial semiconductors based on atomically thin lead iodide crystals. Adv. Mater. 31, 1806562 (2019).

38. Zheng, W. et al. Direct vapor growth of $2 \mathrm{D}$ vertical heterostructures with tunable band alignments and interfacial charge transfer behaviors. Adv. Sci. 6 , 1802204 (2019).

39. Yang, T. et al. Ultrahigh-performance optoelectronics demonstrated in ultrathin perovskite-based vertical semiconductor heterostructures. ACS Nano 13, 7996-8003 (2019)

40. Jiang, Y., Wang, X. \& Pan, A. Properties of excitons and photogenerated charge carriers in metal halide perovskites. Adv. Mater. 31, 1806671 (2019).

41. Zheng, W. et al. Probing and manipulating carrier interlayer diffusion in van der Waals multilayer by constructing type-I heterostructure. Nano Lett. 10, 7217-7225 (2019)

42. Fox, M. Optical Properties of Solids (Oxford University Press, 2010).

43. McCreary, K. M., Currie, M., Hanbicki, A. T., Chuang, H.-J. \& Jonker, B. T. Understanding variations in circularly polarized photoluminescence in monolayer transition metal dichalcogenides. ACS Nano 11, 7988-7994 (2017).

44. Zeng, H., Dai, J., Yao, W., Xiao, D. \& Cui, X. Valley polarization in $\mathrm{MoS}_{2}$ monolayers by optical pumping. Nat. Nanotechnol. 7, 490-493 (2012).

45. Chirvony, V. S. et al. Delayed luminescence in lead halide perovskite nanocrystals. J. Phys. Chem. C 121, 13381-13390 (2017).

\section{Acknowledgements}

We acknowledge financial support from the National Natural Science Foundation of China (Nos. 91850116, 51772084, 51525202, 11774084, and 91833302), the Sino-German Center for Research Promotion (No. GZ1390), the Hunan Provincial Natural Science Foundation of China (No. 2018RS3051, 2019XK2001), and the Project of Educational Commission of Hunan Province of China (No. 18A003).

\section{Author contributions}

X.W. conceived the original idea. D.Z. performed all experiments with assistance from M.H. and L.H. Y.L. and D.Z. prepared the samples for the experiments. A.Z. and M.C performed the band structure calculation. X.W., D.Z., Z.Z., S.C., and W.Z. analyzed the experimental data and performed theoretical simulations. D.Z. and X.W. wrote the manuscript with inputs from Q.T, K.B. and A.M. X.W. and A.P. supervised the work. All authors contributed to the discussion of results and commented on the manuscript.

\section{Competing interests}

The authors declare no competing interests.

\section{Additional information}

Supplementary information is available for this paper at https://doi.org/10.1038/s41467 020-18307-w.

Correspondence and requests for materials should be addressed to X.W. or A.P.

Peer review information Nature Communications thanks the anonymous reviewer(s) for their contribution to the peer review of this work.

Reprints and permission information is available at http://www.nature.com/reprints

Publisher's note Springer Nature remains neutral with regard to jurisdictional claims in published maps and institutional affiliations. 
(c) (i) Open Access This article is licensed under a Creative Commons Attribution 4.0 International License, which permits use, sharing, adaptation, distribution and reproduction in any medium or format, as long as you give appropriate credit to the original author(s) and the source, provide a link to the Creative Commons license, and indicate if changes were made. The images or other third party material in this article are included in the article's Creative Commons license, unless indicated otherwise in a credit line to the material. If material is not included in the article's Creative Commons license and your intended use is not permitted by statutory regulation or exceeds the permitted use, you will need to obtain permission directly from the copyright holder. To view a copy of this license, visit http://creativecommons.org/ licenses/by/4.0/.

(C) The Author(s) 2020 